\title{
SIMULTANEITY, CONVENTION, AND GAUGE FREEDOM
}

\author{
ROBERT RYNASIEWICZ \\ Department of Philosophy \\ Johns Hopkins University \\ Baltimore, MD 21218 \\ ryno@lorentz.phl.jhu.edu
}

\begin{abstract}
As is well know from Einstein (1905) the choice of a criterion for distant simultaneity is equivalent to stipulating one-way speeds for the transit of light. It is shown that any choice of non-standard synchrony is equivalent to a Lorentz local time boost. From this and considerations from the hole argument, it follows that there is a non-trivial sense in which distant simultaneity is conventional, at least to the extent that the "gauge freedom" arising in the hole argument is non-trivial.
\end{abstract}

\section{INTRODUCTION}

At the beginning of the 'Kinematischer Teil' of Zur Elektrodynamik bewegter Körper, Einstein intimates that the method he suggests for synchronizing distant, stationary clocks is arbitrary, and thus that there is no fact of the matter as to the one-way speed of light on which it is based (Einstein 1905). The universal constant $c$ is given by experience only as the average round trip speed of light. His early philosophical admirers, Schlick (1917) and Reichenbach (1924, 1928), highlighted the conventional character of distant simultaneity as the keystone to the theory. Later, Grünbaum $(1963,1973)$ argued for the need of a conventional definition based on the causal theory of time. These defenders of Einstein took as their principal opponents those who, pace relativity, maintained that distant simultaneity is absolute.

More recently it has been claimed that, although simultaneity is relative to the choice of inertial frame, nonetheless, given an inertial frame, it is not a matter of convention as to whether two events are simultaneous. It is a matter of fact that the one-way speed of light (in vacuo) is always $c$. This view has been especially fueled by Malament's (1977) result the only non-trivial simultaneity relation definable from the causal structure of Minkowski spacetime has for its equivalence classes the hyperplanes orthogonal to the world lines at rest in the inertial frame in question. Norton (1992) sees this as "one of the most dramatic reversals in debates in the philosophy of space and time" (p. 194). Despite this, Grünbaum 
(2009) and other defenders of the conventionality thesis have not been persuaded. ${ }^{1}$ As Allen Janis puts it in his Stanford Encyclopedia entry (2010) on the topic, "The debate about conventionality of simultaneity seems far from settled, although some proponents on both sides of the argument might disagree with that statement."

In what follows, I offer a new take on the problem which has the merit of nailing the exact sense in which simultaneity is conventional. It is conventional in precisely the same sense in which the gauge freedom that arises in the general theory of relativity makes the choice between diffeomorphically related models conventional. In fact, the account given here can be seen as nothing more than a variant of the hole argument. If the rationale is not Einstein's of 1905, it is at least a rationale Einstein could have, and maybe should have given after 1915. Nonetheless, the Problemstellung is taken from the Einstein of 1905.

\section{Initial Conditions}

Recall the very first steps Einstein takes in developing the kinematics of special relativity in the first section of Zur Elektrodynamik bewegter Körper (Einstein 1905). Initially, we are granted an inertial frame $F$, "a coordinate frame in which the equations of Newtonian mechanics hold." Think of this as just an inertial fibration of an $\Re^{4}$ manifold. Next, Einstein specifies the use of rigid rods and Euclidean geometry in order to assign position coordinates to a material point at rest. Since we want to do without coordinates to the extent we can, think of this as the assignment of a Euclidean metric $h_{a b}$ on the space $F$ of fibres. Finally, Einstein populates his inertial frame with stationary clocks of identical constitution [von genau derselben Beschaffenheit]. Thus, we have a temporal metric $\theta_{A}$ on each fibre $A \in F$.

Granted this much, which we will call the Einstein frame-structure, it remains impossible to describe a material point in motion. As Einstein puts it, we so far have defined, for fibres $A$ and $B$, only an ' $A$-time' and a ' $B$-time', but no 'common time' for $A$ and $B$. Famously, such a 'common time' arises by stipulating by definition $[$ durch Definition festsetzt, emphasis Einstein's] that it takes the same amount of time for light to travel from $A$ to $B$ as from $B$ to $A .^{2}$

In slightly more detail, suppose at the ' $A$-time' $t_{A}$ a light ray is sent from $A$ to $B$, at the $B$-time $t_{B}$ is reflected from $B$ towards $A$, and at the $A$-time $t_{A}^{\prime}$ arrives back at $A$. Then the two clocks run synchronously by definition [definitionsgemäß] if

$$
t_{B}-t_{A}=t_{A}^{\prime}-t_{B}
$$

Presumably, though, this is an arbitrary choice. It could have been stipulated, alternatively, that it takes twice the time for light to go from $A$ to $B$ as from $B$ to $A$, or half the time,

\footnotetext{
${ }^{1}$ For a bibliography up until 2006, see Jammer (2006).

${ }^{2}$ Einstein (1917) reads "[whether it takes the same amount of time] is in truth no assumption or hypothesis about the physical nature of light, but a stipulation that I can make at my discretion in order to arrive at a definition of simultaneity." (p. 15, translation mine, emphasis in original)
} 
or any given ratio you will. That is, in the equation

$$
\frac{t_{B}-t_{A}}{t_{A}^{\prime}-t_{B}}=\alpha
$$

any choice of $\alpha \in \Re^{+}$is just as legitimate as any other. There is no fact of the matter to be captured, at least according to Einstein. And presumably, Einstein's only reason for choosing $\alpha=1$ is descriptive simplicity.

If we set

$$
\varepsilon:=\frac{\alpha}{1+\alpha}
$$

we retrieve the familiar Reichenbach equation

$$
t_{B}=t_{A}+\varepsilon\left(t_{A}^{\prime}-t_{A}\right) \text {. }
$$

For Einstein's stipulation of $\alpha=1$, equation (2) implies $\varepsilon=1 / 2$, and since $\alpha$ must be positive $\varepsilon$ is constrained to lie in the open interval $(0,1)$. All this is familiar. But rather than following the tradition that begins with Reichenbach, I would like to set out along a slightly different path. ${ }^{3}$

\section{From Times of Transit to Local Time and Back}

Suppose we have two pairs of clocks at $A$ and $B$, one pair synchronized according to the Einstein stipulation $\alpha=1$, the other pair synchronized non-standardly. (If you like, for the sake of definiteness, take $\alpha>1$. But nothing hinges on this in what follows.) How do the two systems of 'common' time, let us say $t$ for the standard and $\tau$ for the non-standard, compare with one another?

When light is emitted from $A$, we can, without loss of generality, set $t_{A}=0=\tau_{A}$. Let $d$ be the distance from $A$ to $B$. Since the two clocks at $A$ are supposed to be of identical constitution, it follows that $t_{A}^{\prime}=2 d / c=\tau_{A}^{\prime}$. So the question is, how do the clocks at $B$ compare? Plugging these values for $t$-time at $A$ into equation (1) for $\alpha=1$ yields $t_{B}=d / c$, as expected. Doing the like for $\tau$-time gives

or

$$
\frac{\tau_{B}-0}{(2 d / c)-\tau_{B}}=\alpha
$$

Thus,

$$
\tau_{B}=\frac{2 \alpha}{\alpha+1} \cdot \frac{d}{c}
$$

$$
\tau_{B}-t_{B}=\frac{\alpha-1}{\alpha+1} \cdot \frac{d}{c}
$$

Now let us suppose that the interval between $A$ and $B$ lies along the positive $x$-axis with $A$ at the origin. Suppose that $\alpha \neq 0$ only along the $x$-axis, and furthermore suppose that $\alpha$ is not a function of $x$. If we set

$$
v:=\left(\frac{1-\alpha}{1+\alpha}\right) c
$$

\footnotetext{
${ }^{3}$ For a reevaluation of Reichenbach's conventionality thesis, see (Rynasiewicz 2003).
} 
and now let $x$ be the distance between the clocks, then

$$
\tau_{B}=t_{B}-v x / c^{2}
$$

This is Lorentz's equation for local time in the Versuch (1895).

Conversely, a choice of $v$ in the equation for local time determines a unique value

$$
\alpha=\left(\frac{1-v / c}{1+v / c}\right)
$$

in the fundamental equation

$$
\frac{t_{B}-t_{A}}{t_{A}^{\prime}-t_{B}}=\alpha
$$

Thus, any choice of simultaneity criterion is equivalent to the choice of a velocity in Lorentz's equation for local time. I'm inclined to think that this equivalence is too elementary for Einstein to have missed and that there is an historical story to be told here. But that is not the concern at present. Rather, local time affords us a standard point mapping whose action on Minkowski spacetime is equivalent to stipulating a different simultaneity criterion.

\section{The Action of Local Time}

Let $\mathfrak{M}=\left\langle E^{4}, \eta\right\rangle$ be a Minkowski space-time, i.e., $E^{4}$ is a manifold diffeomorphic to $\Re^{4}$ and $\eta$ is a Minkowski metric on $E^{4}$. Choose some inertial fibration $F$ of $\mathfrak{M}$, and let $\mathcal{C}=(t, x, y, z$,$) be a global chart adapted to F$ in which

$$
\eta_{\mu \nu}=\left(\begin{array}{cccc}
c^{2} & 0 & 0 & 0 \\
0 & -1 & 0 & 0 \\
0 & 0 & -1 & 0 \\
0 & 0 & 0 & -1
\end{array}\right)
$$

Finally, let $d: E^{4} \rightarrow E^{4}$ be a diffeomorphism such that for all $p \in E^{4}$,

$$
\begin{aligned}
t(d p) & =t(p)-\left(v / c^{2}\right) x(p) \\
x(d p) & =x(p) \\
y(d p) & =y(p) \\
z(d p) & =z(p) .
\end{aligned}
$$

The action of $d$ on various geometrical objects is as follows. First of all, $d$ maps each fibre $A \in F$ to itself. For these are world-lines with constant coordinates independent of $t$. Second, since each fibre is mapped to itself, $d$ maps $h_{a b}$ to itself. Third, for each fibre $A \in F$, the temporal metric $\theta_{A}$ is mapped to itself. For $\theta_{A}$ registers only time differences, and $d$ does not shrink or expand time intervals. In other words, $d$ preserves all of the Einstein frame-structure. 
However, $d$ is not an isometry of $\mathfrak{M}$. It does not preserve the Minkowksi metric. Specifically,

$$
d^{*} \eta_{\mu \nu}=\left(\begin{array}{cccc}
c^{2} & 0 & 0 & v \\
0 & -1 & 0 & 0 \\
0 & 0 & -1 & 0 \\
v & 0 & 0 & -\left(1-v^{2} / c^{2}\right)
\end{array}\right)
$$

Not only is $d$ not an isometry, it is not a conformal mapping. It does not map light cones to light cones. The image of the forward light cone at the origin, which satisfies the equation

$$
t= \pm \frac{\sqrt{x^{2}+y^{2}+z^{2}}}{c}
$$

maps to a cone tilted to the left along the $x$-axis, satisfying the equation

$$
t=\frac{v x}{c^{2}} \pm \frac{\sqrt{x^{2}+y^{2}+z^{2}}}{c} .
$$

The degree of tilting is easier to read if one restricts equation (8) to the $x$ - $t$ plane by setting $y=0=z$. Then the equations for the rays in the positive and negative directions are, respectively,

and

$$
t=(1+v / c)\left(\frac{x}{c}\right)
$$

$$
t=(1-v / c)\left(-\frac{x}{c}\right) .
$$

The tilting of the light cone is the result of choosing anisotropic speeds for light propagation along the $x$-axis corresponding to the local time transformation (5).

What we have seen so far is that the Einstein frame-structure underdetermines the conformal structure of Minkowski spacetime. Does this vindicate Einstein's claim to the effect that there is no fact of the matter as to the ratio of the time out to the time back? After all, the two Minkowski spacetimes $\mathfrak{M}$ and $d^{*} \mathfrak{M}=\left\langle E^{4}, d^{*} \eta\right\rangle$ share the same Einstein framestructure, but disagree on the speed of light along the $x$-axis. Is an arbitrary, stipulative definition required to pick out one of these two isometric ${ }^{4}$ geometries over the other?

\section{Interpretive Options}

The options may become clearer if we pose the question in terms familiar from the so-called hole argument. That argument proceeds by applying a diffeomorphism $d$ to a relativistic spacetime $\mathfrak{A}=\langle M, g\rangle$, where $M$ is an arbitrary four-dimensional manifold, $g$ is a Lorentzian metric, and $d$ is the identity map up through some given time but diverges from the identity on some remaining region $R \subseteq M$ so that $d^{*} g \neq g$ in $R$. One has at hand two interpretive options.

Model Literalism: $d^{*} \mathfrak{A}={ }_{d f}\left\langle M, d^{*} g\right\rangle$ represents a physical situation distinct from that represented by $\mathfrak{A}$.

\footnotetext{
${ }^{4}$ The isometry in question is just $d$. For $d$ is a diffeomorphism of $E^{4}$, and, trivially, $d$ maps $\eta$ to $d^{*} \eta$. However, $d$ is not an isometry (automorphism) of $\left\langle E^{4}, \eta\right\rangle$ since $d^{*} \eta \neq \eta$ as shown above.
} 
Gauge Equivalence: $d^{*} \mathfrak{A}$ represents the same physical situation as that represented by $\mathfrak{A}$.

Opting for model literalism saddles one to a commitment to indeterminism-no-matter-what in classical field theory. For then we have two worlds that agree on the entire past prior to $R$, but disagree on $R$. And the construction is completely general and applicable to any relativistic spacetime. Thus, gauge equivalence is the reasonable interpretive option, not just for instances where radical local indeterminism threatens, but for pairs of models related by any diffeomorphism whatsoever. This is standard practice for working relativists.

Now the case of distinct Minkowski spacetimes related by a diffeomorphism is just a special case of relativistic spacetimes in general. Indeed, in Einstein's original hole argument [Lochbetrachtung], the region $R$ is a "matter hole" in which the stress-energy tensor vanishes identically and the metrics inside $R$ are Minkowskian. Gauge equivalence in our case means that a pair of globally Minkowski metrics related by a local time transformation represent one and the same situation. Although they disagree on the layout of the light cones on $E^{4}$ and hence on the one-way speeds of light along the $x$-axis of chart $\mathcal{C}$, they nonetheless represent the same factual situation. Thus, on gauge equivalence, this apparent difference is merely a formal difference - a difference in representation, not in fact. Hence, the choice of one-way speeds of light is an arbitrary conventional choice.

It may be instructive to see what happens if one opts for model literalism. Suppose the world is as special relativity portrays it. Then there arises a factual question as to whether $\mathfrak{M}$ or $d^{*} \mathfrak{M}$ (or some other of the continuum of Minkowski spacetimes on the manifold $E^{4}$ ) correctly portrays the actual world. How might we find out which? You might think this is just a matter of following how the light cones physically get traced out on $E^{4}$. In order to trace them out, though, we need some way of identifying which point is which. Why not just rely on the chart $\mathcal{C}=(t, x, y, z)$ for this? Well, there is a complete symmetry between $\mathfrak{M}$ and $d^{*} \mathfrak{M}$. In chart $\mathcal{C}$, model $\mathfrak{M}$ represents the choice of standard simulaneity while $d^{*} \mathfrak{M}$ represents a non-standard choice, viz., one in which $\alpha=(1-v / c) /(1+v / c)$. But in chart $\mathcal{C}^{\prime}=\left(t-v x / c^{2}, x, y, z\right)$ it is $d^{*} \mathfrak{M}$ that represents the choice of standard simultaneity, while $\mathfrak{M}$ represents a non-standard choice with $\alpha=(1+v / c) /(1-v / c)$. Unless we are given either $\mathfrak{M}$ or $d^{*} \mathfrak{M}$ to begin with, we cannot tell whether we are using chart $\mathcal{C}$ or $\mathcal{C}^{\prime}$. And being granted $\mathfrak{M}$ or $d^{*} \mathfrak{M}$ at the outset simply begs the question. All that we would know is that there is some chart in which the speed of light is isotopic, although we can never know which. It would then be a cosmic coincidence if the choice $\alpha=1$ in chart $(t, x, y, z)$ selected the true one-way speeds of light.

\section{Familiar Objections to Simultaneity by Convention}

Having seen how the conventionality of simultaneity arises as a corollary to the hole argument, it is instructive to review the usual objections to the conventionality of simultaneity to see just where they miss the mark.

6.1. Trivial Coordinate Freedom. Both Friedman (1983) and Norton (1992) have charged that the conventionality thesis becomes trivial if all that it shows is that it is possible to 
use a coordinate chart corresponding to a non-standard choice of simultaneity. To quote from Norton (1992),

Winnie (1970) showed that we can generalize a standard coordinate system of special relativity to a new coordinate system with time coordinate $t_{\epsilon}$ in such a way that events with equal $t_{\epsilon}$ are judged simultaneous by some $\epsilon$-criterion. It is sometimes thought that this fact by itself is sufficient to vindicate the conventionalist claim. This is obviously false since all that has been shown is that we can extend the covariance of the theory so that it can use $t_{\epsilon}$ coordinate systems. We have seen that it is possible to extend the covariance of the theory even further to general covariance, which allows arbitrary coordinate systems. Indeed we have seen that we can give generally covariant formulations of every spacetime theory considered so far. If we can automatically read the $t$ coordinate of any of these formulations as giving a criterion of simultaneity, then we could vindicate the strangest of simultaneity relations, including nonstandard simultaneity relations even in Newtonian spacetimes. What is needed is some independent means of arguing that the $t$ coordinate of a given formulation does represent a possible simultaneity relation, such as the causal theory of time seeks to provide for $t_{\epsilon}$. (p. 222)

We have not simply switched from a coordinate chart $(t, x, y, z)$ in which the simultaneity relation is standard to a chart $\left(t-v x / c^{2}, x, y, z\right)$ in which that relation is nonstandard. Rather we have used that transformation to induce an active point mapping of the Minkowski spacetime to a new Minkowski spacetime in which the simultaneity relation is standard in the new chart $\left(t-v x / c^{2}, x, y, z\right)$. The indiscernability of the two (isomorphic) spacetimes gives us an independent reason for arguing that there are different possible simultaneity relations for a given Einstein frame-structure on the manifold.

The quote, however, raises another concern. Can't we also use local time boosts in Newtonian spacetimes to generate non-standard simultaneity relations? The answer to this question depends on the physics we imagine in Newtonian spacetime. If light remains, as Reichenbach called it, a first signal, i.e., an upper bound on causal propagation, then we should expect the possibility of non-standard simultaneity relations. This replicates the historical situation at the end of the nineteenth century. But if the physics includes, say, Newtonian gravitation, then not. The reason is that then more is built into the original frame-structure by relying on instantaneous gravitational signaling or clock transport. And this addition to the frame-structure (which turns out to be nothing other than absolute simultaneity) is not preserved under local time boosts. This is very different from simply grafting coordinate charts onto the geometry.

6.2. Extra, Gratuitous Structure. It has seemed to many that if the speed of light is not isotropic, then there must exist some additional, symmetry breaking spacetime structure to define a preferred direction along which the speed of light is anisotropic. This is what Friedman (1983) has in mind when he writes, 
$\ldots$ it is clear that if we wish to employ a nonstandard $(\epsilon \neq 1 / 2)$ simultaneityrelation-for- $\sigma$ we must add further structure to Minkowski space-time. For example, to employ our "tilted" simultaneity relation we have to pick out a distinguished spatio-temporal orientation that is not itself definable from the ("isotropic") metric $g$. This additional structure has no explanatory power, however, and no useful purpose is served by introducing it into Minkowski space-time. Hence, the methodological principle of parsimony favors the choice of Minkowski space-time, with its "built-in" standard simultaneity, over Minkowski space-time plus any additional nonstandard simultaneity. (p. 312)

This would be true if we attempted to define simultaneity only after having fixed the light cone structure on spacetime. But as we have seen, the problem of defining simultaneity is equivalent to the problem of determining how to draw the light cones in spacetime after having been given an Einstein frame-structure on the spacetime, i.e., an inertial frame with Euclidean geometry and an ensemble of identically constituted clocks at rest in that frame. The speed of light is isotropic or anisotropic only with respect to a coordinate chart adapted to that frame. This coordinate chart is not part of the intrinsic structure of spacetime. As we have seen, if the speed of light is anisotropic in one coordinate chart, there is a chart related to it by a local time transformation in which the speed of light is isotropic.

6.3. Malament's Result. By far the most influential objection has been based on a result due to David Malament (1977). Again, quoting from Norton (1992),

We return to the conventionality of simultaneity ... to see one of the most dramatic reversals in debates in the philosophy of space and time. David Malament has recently derived a theorem in special relativity which, he urges, shows that the causal relations of special relativity do not leave the simultaneity relation underdetermined and thus the relation cannot be set conventionally within the causal theory of time. He shows that the only nontrivial simultaneity relation definable in terms of the causal relations of special relativity is the familiar standard simultaneity relation of $\epsilon=1 / 2$.

(p. 194)

Malament's result, however, has simply been misapplied to the problem of the conventionality of simultaneity. For the result presupposes that we first fix the light cone structure, i.e., the one-way speeds of light, and then ask what simultaneity relations are definable from this. And obviously the standard relation is uniquely definable from this. The original problem, however, was whether there is a unique way to draw in the light cones, given the Einstein frame-structure. If we draw the cones in with anisotropic one-way velocities, that also defines a unique, but non-standard simultaneity relation.

It may seem there is a paradox here. For doesn't the causal structure fix the light cone structure? Yes, it does. However, the Einstein frame structure does not fix the causal connectability relation $\kappa$ on the manifold. In his (1977), Malement set up his result by using specifically the manifold $\Re^{4}$. In a footnote he observes: "In a suitable second-order 
formal language containing a single two-place predicate symbol (for $\kappa$ ) one can formulate a finite set of axioms every model of which is isomorphic to $\left(R^{4}, \kappa\right)$ " (fn 2 , p. 300). What do the other models isomorphic to $\left(R^{4}, \kappa\right)$ look like? Well, some of them can be on a manifold that is not $R^{4}$ but diffeomorphic to $R^{4}$. That is one possibility. But what about other isomorphic models on $R^{4}$ ? There are as many of them as there are diffeomorphisms of $R^{4}$ modulo automorphisms. For let $d$ be a diffeomorphism that is not a symmetry. This induces a relation $d^{*} \kappa$ such that $p$ is $d^{*} \kappa$ related to $q$ if and only if $d^{-1}(p)$ is $\kappa$ related to $d^{-1}(q)$. Thus, one can have many different causal connectability relations on the same manifold, all of which represent one and the same physical situation according to the policy of "gauge equivalence." The situation is no different than the initial value problem in general relativity. The metric, including the conformal and causal structure, is determined from the given Cauchy surface only up to a choice of gauge. The synchronization problem is nothing more than a fixing of gauge for flat relativistic spacetime.

If you say that synchronization and the (one-way) speed of light is conventional, does this not mean then that causal connectability is also conventional, and isn't that a reductio of your thesis? No. The proper thing to say is not that causal connectability is conventional. For the physical facts expressed by the equivalence class of isomorphic models is certainly not a matter of convention. However, the choice between the many different representations of causal connectability is conventional. And so it goes with simultaneity. There are certainly things about the conformal structure of Minkowski spacetime that are not conventional (e.g., that it's flat). But the choice between the many different representations of conformal structure on the manifold certainly is conventional, and what differs between those various representations is the one-way speed of light in a given chart adapted to the Einstein frame-structure. That is the exact sense in which simultaneity is conventional.

\section{Conclusion}

In what precedes I have offered a very different rationale for taking seriously the conventionality of simultaneity than any that have appeared before. Choices of different standards of simultaneity are equivalent to active boosts under local time. Active boosts under local time preserve the antecedently given Einstein frame-structure but do not preserve light cone structure, and hence one-way speeds of light. Yet models related by local time boosts represent the same physical situation. Thus, the one-say speed of light does not have a unique representation in spacetime.

While I tend to believe that Einstein was aware of the connection between local time and the choice of simultaneity criterion in May or June of 1905, and even though he ultimately resolved his Lochbetractung with gauge equivalence in 1915, he never, to my knowledge, put the two together, even though the opportunity presented itself in 1916 when he drafted his popular exposition of relativity (Einstein 1917). There he makes sufficient todo about the conventionality of simultaneity (the reader should not read on without understanding it), but the rationale is of an operationalist sort: the concept exists for the physicist only if it can be determined in concrete cases whether or not the concept applies. Nonetheless, gauge equivalence is just the cure for these verificationist impulses. 
Two issues remain. The first is, why are we restricted at the outset to Einstein framestructure? The easy answer is that that is all that Einstein gives us, and that my purpose here is a limited one: to reconstruct rationally the sense in which Einstein claimed that simultaneity is conventional. But the issue can be pressed further. Did Einstein take into consideration all means available for fixing coordinate charts? I believe that if one considers the totality of what he understood to be granted in constructing charts in Newtonian spacetime, Einstein frame-structure is all that can be rightly carried over to the relativistic setting. It's fairly easy to predict that anything richer that suffices to fix conformal structure will have a convention built in equivalent to a choice of one-way light speeds.

The second issue is whether the sense given here in which simultaneity is conventional is non-trivial. Now there are only examples of triviality but no criteria for triviality. Clearly, it's not trivial semantic conventionality. Nor is it trivial coordinate freedom. As indicated earlier, it is basically a corollary of the hole argument, and so is no more trivial than the recognition of gauge equivalence in general relativity. If the latter strikes you as trivial, I suggest it's only because you've run through the hole argument so many times that you now find it humdrum. Good. If you find the conventionality of simultaneity similarly unexciting, it's only because it is now so obvious to you.

Acknowledgements. This work was sponsored by NSF grant no. SES-0725585. Talks based on these ideas were previously given at Minnesota, Oxford, Utrecht, Pittsburgh, and New Directions in the Foundations of Physics. Especial thanks to Allen Janis, John Norton, David Malament, Bas van Fraassen, Marius Stan, Shane Steinert-Threlkheld, and Michel Janssen for helpful comments on previous drafts.

\section{REFERENCES}

[Einstein(1905)] Einstein, Albert. "Zur Elektrodynamik bewegter Körper." Annalen der Physik 17: (1905) 891-921. Reprinted as "On the Electrodynamics of Moving Bodies"," in H.A. Lorentz et. al., The Principle of Relativity, New York: Dover, 37-65.

[Einstein(1917)] —. Über die spezielle und die allgemeine Relativitätstheorie (Gemeinverständlich). Braunschweig: Fr. Vieweg \& Sohn, 1917. Reprinted in Collected Papers, vol. 6, 421-534.

[Friedman(1983)] Friedman, Michael. Foundations of Space-Time Theories: Relativistic Physics and Philosophy of Science. Princeton: Princeton University Press, 1983.

[Grünbaum(1963)] Grünbaum, Adolph. Philosophical Problems of Space and Time. New York: Alfred A. Knopf, 1963.

[Grünbaum(1973)] _ Philosophical Problems of Space and Time. Boston Studies in the Philosophy of Science, vol, 12. Synthese Library. Dordrecht and Boston: Reidel, 1973, 2nd enlarged edition.

[Grünbaum(2010)] _ _ "David Malament and the Conventionality of Simultaneity: A Reply." Foundations of Physics 40: (2010) 1285-1297.

[Jammer(2006)] Jammer, Max. Concepts of Simultaneity: From Antiquity to Einstein and Beyond. Baltimore: Johns Hopkins Press, 2006.

[Janis(2010)] Janis, Allen. "Conventionality of Simultaneity." In Stanford Encyclopedia of Philosophy, edited by Edward N. Zalta. 2010. http://plato.stanford.edu/entries/spacetime-convensimul/.

[Lorentz(1895)] Lorentz, H.A. Versuch einer Theorie der electrischen und optischen Erscheinungen in bewegten Körpern. Leiden: E.J. Brill, 1895.

[Malament(1977)] Malament, David. "Causal Theories of Time and the Conventionality of Simultaneity." Nô̂s 11: (1977) 293-300. 
[Norton(1992)] Norton, John. "Philosophy of Space and Time." In Introduction to the Philosophy of Science, edited by Merrilee Salmon et. al., Englewood Cliffs, N.J.: Prentice Hall, 1992, 179-231.

[Reichenbach(1924)] Reichenbach, Hans. Axiomatik der relativistischen Raum-Zeit-Lehre. Braunschweig: Fr. Vieweg \& Sohn, 1924.

[Reichenbach(1928)] —. Philosophie der Raum-Zeit-Lehre. Berlin and Leipzig: Walter de Gruyter \& Co., 1928.

[Rynasiewicz(2003)] Rynasiewicz, Robert. "Reichenbach's $\varepsilon$-Definition of Simultaneity in Historical and Philosophical Perspective." In The Vienna Circle and Logical Empiricism, edited by Friedrich Stadler, Dordrecht: Kluwer, 2003, 121-129.

[Schlick(1917)] Schlick, Moritz. Raum und Zeit in der gegenwärtigen Physik. Berlin: Julius Springer, 1917.

[Winnie(1970)] Winnie, John. "Special Relativity without One-Way Velocity Assumptions." Philosophy of Science 37: (1970) 81-99, 223-238. 